\title{
Úlceras por pressão em pacientes internados em unidades de terapia intensiva: revisão integrativa da literatura
}

\author{
Pressure ulcers in patients in intensive care units: integrative literature review
}

\author{
Antonia Almeida Araújo ${ }^{a}$, Ariane Gomes dos Santos ${ }^{b}$ \\ a Acadêmica de Enfermagem do Instituto de Ensino Superior Múltiplo do Maranhão (IESM). \\ b Enfermeira. Mestre em Ciências e Saúde. Professora do Curso de Enfermagem do IESM. Enfermeira do Instituto Federal de Educação, Ciência e Tecnologia do Piauí (IFPI).
}

Fontes de financiamento: Instituto de Ensino Superior Múltiplo do Maranhão - IESM, por meio do Programa Institucional de Bolsas de Iniciação Científica (PIBIC).

RESUMO

Objetivo: Analisar a produção científica acerca de úlcera por pressão (UPP) em pacientes internados em unidades de terapia intensiva (UTI).

Materiais e Métodos: Trata-se de uma revisão integrativa da literatura. A coleta de dados foi realizada de julho a novembro de 2014, as bases de dados utilizadas foram: Literatura Latino-Americana e do Caribe em Ciências da Saúde (LILACS), National Library of Medicine (NLM-PubMed) e Scientific Eletronic Library OnLine (SCIELO). A amostra final do estudo constituiu-se de 14 artigos.

Resultados: Os conteúdos dos artigos foram divididos em três categorias analíticas: "relação entre a prevenção de UPP e assistência de enfermagem em UTI" (71,4\% dos artigos), "fatores de risco para UPP em pacientes internados em UTI" (64,2\%) e "fatores propulsores de assistência de enfermagem qualificada" (35,7\%). A pesquisa pôde constatar a viabilidade clínica da escala de Braden e a inegável importância da mudança de posicionamento, considerada como a principal ação da equipe de enfermagem na prevenção de UPP.

Conclusões: Pacientes internados em UTI estão mais expostos ao desenvolvimento de UPP, cabe à equipe de enfermagem realizar um cuidado sistematizado e adotar a construção de uma cultura de avaliação orientada por um paradigma educativo, que preconiza um olhar contínuo para a melhoria do serviço prestado. É necessária a realização de educação em saúde e discussões acerca da temática, assim como novas pesquisas que continuem avaliando os riscos associados ao surgimento das UPP.

Palavras-chaves: úlcera por pressão; enfermagem; unidades de terapia intensiva.

Objective: To analyze the scientific production on pressure ulcers (UPP) in patients admitted to intensive care units (ICU).

Materials and Methods: This is an integrative literature review. Data collection was carried out from July to November 2014, the databases used were: Latin American and Caribbean Literature in Health Sciences (LILACS), International Literature in Health Sciences (MEDLINE) and Scientific Electronic Library OnLine (SCIELO). The final study sample consisted of 14 articles and data analysis proceeded descriptively.

Results: The contents of the articles were divided into three analytical categories: "the relationship between the prevention of UPP and nursing care in the ICU" (71.4\% of articles), "risk factors for UPP in ICU patients" (64.2\%) and "assistance thrusters factors of qualified nursing" (35.7\%). The survey was able to establish the clinical viability of the Braden Scale and the undeniable importance of changing position, regarded as the main action of the nursing staff in the prevention of UPP.

Conclusions: ICU patients are more exposed to developing UPP, it is for the nursing staff to perform a systematic care and to adopt a culture of evaluation guided by an educational paradigm, which advocates a continuous look at the improvement of service. It is necessary to carry out health education and discussions on the theme, as well as new research to continue assessing the risks associated with the emergence of UPP.

Keywords: pressure ulcer; nursing; intensive care unit. 


\section{INTRODUÇÃO}

As úlceras por pressão são lesões localizadas da pele e/ ou tecido subjacente, geralmente sobre uma proeminência óssea, em resultado da pressão ou de uma combinação entre esta e forças de torção. Às úlceras por pressão, também estão associados fatores contribuintes e de confusão, cujo papel ainda não se encontra totalmente esclarecido ${ }^{1}$.

O desenvolvimento da úlcera por pressão (UPP) é multifatorial, incluindo fatores internos do indivíduo (idade, morbidades, estado nutricional, hidratação, condições de mobilidade e nível de consciência) e externos (pressão, cisalhamento, fricção e umidade) ${ }^{2,3}$. São consideradas um problema grave, especialmente em pessoas idosas e clientes portadores de doenças crônico-degenerativas. Sua prevalência representa uma significante ameaça aos pacientes com mobilidade prejudicada ou comprometimento da percepção sensorial. Além disso, aumentam a morbimortalidade, o tempo e custo de internação, interferindo na qualidade de vida dos pacientes ${ }^{2}$.

As UPP são, muitas vezes, decorrentes da ausência de execução de normas básicas de segurança do paciente 4 . Desse modo, vale ressaltar o contexto do movimento global pela segurança do paciente que visa promover e apoiar a implementação em diferentes áreas da atenção, organização e gestão de serviços de saúde, por meio da implantação da gestão de risco e de Núcleos de Segurança do Paciente nos estabelecimentos de saúde ${ }^{5}$.

A prevenção de UPP é uma questão que deve ser avaliada, a maioria dos casos de UPP pode ser evitada por meio da identificação dos pacientes em risco e da implantação de estratégias de prevenção confiáveis para todos os pacientes identificados como de risco. A complexidade e a gravidade dos pacientes internados nas Unidades de Terapia Intensiva resultam na necessidade de reavaliação diária do potencial e do risco de desenvolvimento de UPP. A reavaliação diária permite aos profissionais de saúde ajustar suas estratégias de prevenção conforme as demandas do paciente ${ }^{6}$.

A ocorrência de úlcera por pressão merece atenção diferenciada, visto que, constitui um problema de saúde importante, sobretudo considerando o impacto que tem para o paciente, família e sociedade, sendo apontado como um indicador negativo de qualidade assistencial dos serviços de saúde ${ }^{7}$. É sabido que a UPP deve ser considerado um problema de saúde que envolve toda a equipe multiprofissional de uma Unidade de Terapia Intensiva envolvendo, sobretudo, a equipe de Enfermagem, que oferece cuidados contínuos aos pacientes 24 horas por dia. O tratamento individualizado por meio da sistematização da assistência em enfermagem assim como a utilização da prática clínica baseada em evidências pode proporcionar efeitos positivos na qualidade do cuidado ao paciente ${ }^{3,8}$.

Estudos pontuam a importância de pesquisas voltadas para a avaliação de pacientes em estado crítico e métodos que favoreçam a atuação da enfermagem na prevenção de úlceras por pressão, resultando, dessa forma, na melhoria da qualidade da assistência de enfermagem, proporcionando maior segurança para o paciente, especialmente aqueles internados em unidade de terapia intensiva em razão da gravidade de suas condições clínicas $7,9,10$.

Dentre as recomendações gerais para avaliação de risco estruturado, segundo a NPUAP (National Pressure Ulcer Advisory Panel) em 2014, estão: realizar uma avaliação de risco estruturado logo que possível (mas no prazo máximo de oito horas depois admissão) para identificar os indivíduos com risco de desenvolver úlceras por pressão; repetir essa avaliação de acordo com a necessidade do paciente; realizar uma reavaliação,se houver qualquer mudança significativa na condição do indivíduo; incluir uma análise abrangente da pele para verificar quaisquer alterações à pele intacta; documentar todas as avaliações de risco; desenvolver e implementar um plano de prevenção de riscos ${ }^{11}$.

Tais medidas servem para auxiliar o enfermeiro na mensuração do risco e no planejamento da intervenção terapêutica ${ }^{10,12}$. Estudos ressaltam, ainda, as mudanças de posicionamento como a principal intervenção terapêutica de responsabilidade da enfermagem na prevenção de lesões ${ }^{12,13}$.

Nas unidades de terapia intensiva, a demanda de cuidados especializados e o uso de recursos tecnológicos requer muita atenção, uma vez que, a equipe de saúde tem como preocupação principal a estabilização do quadro clínico do paciente. Nesse contexto, seja pela dificuldade de realização de medidas preventivas para preservação da integridade da pele ou pela gravidade do paciente, observase o desenvolvimento de úlceras por pressão em pacientes. Diante de tal constatação, torna-se imprescindível a análise de estudos sobre úlceras por pressão em pacientes internados em unidades de terapia intensiva. Dessa forma, o presente estudo objetivou analisar a produção científica acerca de úlceras por pressão em pacientes internados em unidades de terapia intensiva.

\section{MATERIAIS E MÉTODOS}

Trata-se de uma revisão integrativa da literatura. Apoderando-se de normas claras e de rigor científico, foram consideradas seis fases para a realização deste estudo: identificação do tema e seleção da hipótese 
ou questão de pesquisa para a elaboração da revisão integrativa; estabelecimento de critérios para inclusão e exclusão de estudos/ amostragem ou busca na literatura; definição das informações a serem extraídas dos estudos selecionados/ categorização dos estudos; avaliação dos estudos incluídos na revisão integrativa; interpretação dos resultados; e apresentação da revisão/síntese do conhecimento $^{14}$

A pergunta que norteou a revisão de Literatura foi: Como estão dispostas as produções científicas sobre úlceras por pressão em pacientes internados em unidades de terapia intensiva?

A coleta de dados foi realizada de julho a novembro de 2014 e a busca foi conduzida na Biblioteca Virtual em Saúde (BVS), na qual foram pesquisadas as seguintes bases de dados: Literatura latino-Americana e do Caribe em Ciências Da Saúde (LILACS), National Library of Medicine (NLMPubMed) e Scientific Eletronic Library OnLine (SCIELO). Para a procura dos artigos indexados foram utilizados os descritores: úlcera por pressão/ pressure ulcer, enfermagem/ nursing e unidades de terapia intensiva/intensive care units, todos de acordo com os descritores em Ciências da Saúde BIREME. Para sistematizar a pesquisa foi aplicado o operador booleano "and", encontrou-se nas bases de dados um somatório de 334 artigos (76 LILACS, 214 MEDLINE, 44 SCIELO).

Os critérios de inclusão foram artigos publicados no recorte temporal de 2008 a 2014; redigidos em língua portuguesa, espanhola ou inglesa; e disponibilizados na íntegra na base de dados. Os critérios de exclusão foram artigos repetidos, resenhas, anais de congresso, artigos de opinião, revisões de literatura, artigos de reflexão, editoriais, teses, dissertações e artigos que não abordaram diretamente o tema deste estudo.

Após o levantamento das publicações, os resumos foram lidos e analisados segundo os critérios de inclusão e exclusão preestabelecidos. Após isso, fez-se uma triagem quanto à relevância e à propriedade que responderam ao objetivo deste estudo chegando a uma amostra final de 14 artigos, os quais foram lidos e analisados na íntegra. Para a apuração dos dados elaborou-se um instrumento com as seguintes variáveis: autores, tipo de estudo, amostra, local do estudo e ano de publicação, principais resultados e conclusões. A análise dos artigos procedeu-se de modo descritivo e os resultados foram apresentados em forma de tabelas, divididos nas seguintes categorias analíticas: "relação entre a prevenção de UPP e assistência de enfermagem em UTI", "fatores de risco para UPP em pacientes internados em UTI" e "fatores propulsores de assistência de enfermagem qualificada".

\section{RESULTADOS E DISCUSSÃO}

\section{Caracterização dos estudos}

Os artigos apresentaram diferentes características no que se refere à amostra e ao delineamento metodológico. Com base na Tabela 1, observou-se que dentre os tipos de estudos selecionados encontrou-se cinco $(35,7 \%)$ estudos transversais e nove $(64,3 \%)$ estudos descritivo-exploratórios. $21,4 \%$ e 14,3\% dos artigos foram encontrados na Revista Eletrônica de Enfermagem - Universidade Federal de Goiás, Brasil e Acta Paulista de Enfermagem da Escola Paulista de Enfermagem - EPE da Universidade Federal de São Paulo - UNIFESP, respectivamente. Dos idiomas dos estudos, 92, 8\% publicados em português e 7\% no idioma inglês. Quanto ao perfil dos sujeitos das pesquisas, destacaram-se enfermeiros, técnicos, auxiliares, além de clientes internados em UTI (Tabela 1).

Foi possível observar que quanto ao delineamento metodológico, as pesquisas mostram desenhos observacionais e analíticos buscando uma maior compreensão sobre a prevenção de úlceras por pressão em pacientes internados em unidades de terapia intensiva e a assistência de enfermagem como subsídio para realização de ações em busca de minimizar o problema.

A seguir serão apresentadas as categorias analíticas nas quais se enquadraram os artigos que constituíram a amostra do presente estudo. Vale ressaltar que um mesmo artigo pode ter compreendido mais que uma categoria.

\section{Relação entre a prevenção de UPP e assistência de enfermagem em UTI}

A categoria que abordou a relação entre a prevenção de UPP e assistência de enfermagem estava presente em 10 artigos (71,4\%). A Tabela 2 mostra os principais resultados apresentados por esses artigos $15,16,17,18,19,20,21,22,23,24$.

As UPP representam um problema social, econômico e educacional. O desenvolvimento das mesmas gera impacto na qualidade de vida dos pacientes e de seus familiares, assim como, no aumento nos custos para as instituições hospitalares. Em estudo realizado em UTI de um hospital privado localizado em Natal/RN, as UPP foram diagnosticadas em $50,0 \%$ dos pacientes, destes $75,0 \%$ apresentaram uma úlcera e 25,0\% duas UPP'15.

Outra Pesquisa desenvolvida no Hospital Metropolitano de Urgência e Emergência (HMUE) em Ananindeua/PA evidenciou que 17 (3,6\%) pacientes desenvolveram úlceras por pressão, dos quais $6(35,3 \%)$ na Clínica Cirúrgica 1, $5(29,4 \%)$ na Neuroclínica, e $6(35,3 \%)$ na UTI ${ }^{16}$.

Percebe-se dessa forma, que as úlceras por pressão são um problema de causalidade multifatorial, uma vez que, sua ocorrência é afetada por fatores fisiológicos, microbiológicos, 
sociais, econômicos, educacionais e comportamentais, resultando no aumento da morbimortalidade dos indivíduos ${ }^{25}$. Estratégias de investimento em capacitação profissional, construção e implantação de protocolos precisam ser implementados em busca da melhoria da qualidade da assistência oferecida ${ }^{25,26,27}$.

A equipe multiprofissional tem a responsabilidade de adotar medidas de prevenção para com os indivíduos que apresentem riscos de desenvolver úlcera por pressão. Em estudo relativo às formas de prevenção e de tratamento de úlceras de pressão, realizado com 15 profissionais de uma equipe de saúde, todos indicaram como medida preventiva a mudança de posicionamento, 60\% indicaram a hidratação da pele com óleo, 80 \% colchão caixa de ovo, 40\% coxim e $20 \%$ massagem ${ }^{17}$.

Estudo exploratório-descritivo realizado na UTI de um hospital universitário da região Sul do Brasil, mostrou que o enfermeiro está diretamente ligado à gerência de cuidados dos pacientes internados em UTI, com mobilidade física reduzida e com predisposição para formação de UPP. Durante a sua atuação no ambiente profissional, eles procuram voltar atenção ao trabalho dos técnicos de enfermagem, orientando, estimulando e supervisionando as atividades que são realizadas tanto em relação ao cuidado na prevenção, quanto no tratamento das lesões já desenvolvidas no paciente ${ }^{20}$.

Nota-se que mudança de posicionamento tornou-se a principal ação de responsabilidade da equipe de enfermagem, com o intuito de prevenir as lesões de pele. Além disso, é relevante salientar sobre a importância do cuidado individualizado, uma vez que, possibilita ao enfermeiro realizar o diagnóstico precoce da lesão, planejar suas ações, acompanhar e avaliar a evolução da lesão ${ }^{17,18}$.

Tabela 1. Caracterização da produção científica quanto aos autores, tipo de estudo, amostragem, local, periódico e ano de publicação.

\begin{tabular}{|c|c|c|c|c|c|}
\hline Autor/Referência & Tipo de estudo & Amostra & Local & Periódico & Ano \\
\hline Fernandes et al. ${ }^{41}$ & $\begin{array}{l}\text { Quantitativo com } \\
\text { delineamento descritivo } \\
\text { comparativo }\end{array}$ & $\begin{array}{l}50 \text { Membros da equipe } \\
\text { de enfermagem }\end{array}$ & $\begin{array}{l}\text { Hospital Universitário do } \\
\text { interior Paulista }\end{array}$ & Acta Paul Enferm & 2008 \\
\hline Fernandes et al. ${ }^{15}$ & $\begin{array}{l}\text { Estudo descritivo, com } \\
\text { delineamento longitudinal e } \\
\text { abordagem quantitativa }\end{array}$ & 40 Clientes & $\begin{array}{l}\text { Unidade de terapia intensiva de } \\
\text { um hospital privado localizado } \\
\text { em Natal-RN }\end{array}$ & Rev. Eletr. Enf. & 2008 \\
\hline Martins \& Soares ${ }^{17}$ & $\begin{array}{l}\text { Estudo descritivo exploratório } \\
\text { com abordagem qualitativa }\end{array}$ & $\begin{array}{l}15 \text { Membros da equipe } \\
\text { de enfermagem }\end{array}$ & $\begin{array}{l}\text { Hospital de médio porte no } \\
\text { interior de Minas Gerais }\end{array}$ & CogitareEnferm & 2008 \\
\hline Cremasco et al. ${ }^{20}$ & Transversal & 74 Clientes & $\begin{array}{l}\text { Unidade de terapia intensiva } \\
\text { de hospital universitário de } \\
\text { nível terciário localizado no } \\
\text { Município de São Paulo-SP }\end{array}$ & Acta Paul Enferm & 2009 \\
\hline Rangel \& Caliri ${ }^{19}$ & Transversal & 25 enfermeiros & $\begin{array}{l}\text { Hospital geral, interior do } \\
\text { estado de São Paulo }\end{array}$ & Rev. Eletr. Enf & 2009 \\
\hline Miyazaki et al. ${ }^{35}$ & $\begin{array}{l}\text { Quantitativo com } \\
\text { delineamento descritivo- } \\
\text { exploratório }\end{array}$ & $\begin{array}{l}386 \text { Membros da } \\
\text { equipe de enfermagem }\end{array}$ & $\begin{array}{l}\text { Hospital de nível terciário do } \\
\text { Interior Paulista }\end{array}$ & $\begin{array}{l}\text { Rev. Latino-Am. } \\
\text { Enfermagem }\end{array}$ & 2010 \\
\hline Gomes et al. ${ }^{23}$ & Seccional Analítico & 142 Clientes & $\begin{array}{l}15 \text { Hospitais públicos e privados } \\
\text { de Belo Horizonte-MG }\end{array}$ & Rev Esc. Enf. USP & 2010 \\
\hline Silva et al. ${ }^{22}$ & Estudo transversal, descritivo & 21 Clientes & $\begin{array}{l}\text { Unidade terapia intensiva de } \\
\text { um Hospital público extraporte } \\
\text { da cidade do Recife-PE }\end{array}$ & Rer. Bras TerIntensiva & 2010 \\
\hline Soares et al. ${ }^{16}$ & $\begin{array}{l}\text { Estudo do tipo observacional, } \\
\text { longitudinal, prospectivo }\end{array}$ & 474 pacientes & $\begin{array}{l}\text { Hospital Metropolitano } \\
\text { de Urgência e Emergência } \\
\text { (HMUE) em Ananindeua-PA }\end{array}$ & Rev. Bras.,Cir. Plást & 2011 \\
\hline Araújo et al. ${ }^{34}$ & $\begin{array}{l}\text { Estudo transversal com } \\
\text { abordagem quantitativa }\end{array}$ & 63 indivíduos & $\begin{array}{l}\text { Unidade de terapia intensiva, de } \\
\text { hospital privado de Fortaleza-CE }\end{array}$ & Rev. enferm. UERJ & 2011 \\
\hline Silva et al. ${ }^{21}$ & $\begin{array}{l}\text { Estudo prospectivo, com } \\
\text { análise quantitativa }\end{array}$ & 189 pacientes & $\begin{array}{l}\text { Hospital de nível terciário, } \\
\text { público, de ensino, localizado } \\
\text { no município de Uberaba-MG }\end{array}$ & Rev. Eletr. Enf. & 2011 \\
\hline Stein et al. ${ }^{18}$ & $\begin{array}{l}\text { Descritivo-exploratório com } \\
\text { abordagem qualitativa }\end{array}$ & 8 enfermeiros & $\begin{array}{l}\text { Unidade de terapia intensiva } \\
\text { de um Hospital Universitário da } \\
\text { região sul do Brasil }\end{array}$ & $\begin{array}{l}\text { Rev. pesqu.: cuid. } \\
\text { Fundam }\end{array}$ & 2012 \\
\hline Silva et al. ${ }^{13}$ & $\begin{array}{l}\text { Estudo descritivo, longitudinal, } \\
\text { de abordagem quantitativa }\end{array}$ & 57 pacientes & $\begin{array}{l}\text { UTI de hospital público de } \\
\text { médio porte, de João Pessoa-PB }\end{array}$ & Rev. Rene & 2013 \\
\hline Dantas et al. ${ }^{24}$ & $\begin{array}{l}\text { Estudo descritivo com } \\
\text { abordagem qualitativa }\end{array}$ & 13 enfermeiros & $\begin{array}{l}\text { Hospital Universitário no } \\
\text { nordeste do Brasil }\end{array}$ & J. res.: Fundam. Care & 2014 \\
\hline
\end{tabular}


Tabela 2. Principais resultados encontrados em estudos que contemplaram a categoria: "relação entre prevenção de UPP e assistência de enfermagem em UTI".

\begin{tabular}{|c|c|c|}
\hline Autor/Referência & Título & Principais resultados \\
\hline Martins \& Soares ${ }^{17}$ & $\begin{array}{l}\text { Conhecimento sobre prevenção e tratamento de } \\
\text { úlceras de pressão entre trabalhadores de enfermagem } \\
\text { em um hospital de Minas Gerais }\end{array}$ & $\begin{array}{l}\text { Como medidas preventivas, os resultados indicaram a mudança de } \\
\text { decúbito }(100 \%) \text {; hidratação da pele com óleo }(60 \%) \text {; colchão caixa } \\
\text { de ovo }(80 \%) \text {; coxim }(40 \%) \text {; massagem }(20 \%) \text {. Para tratamento foram } \\
\text { citadas coberturas, medicamentos e soluções. Quanto à competência dos } \\
\text { profissionais } 33,3 \% \text { disseram seguir prescrição médica e nenhum citou a } \\
\text { prescrição do enfermeiro. }\end{array}$ \\
\hline Cremasco et al. ${ }^{20}$ & $\begin{array}{l}\text { Úlcera por pressão: risco e gravidade do paciente e } \\
\text { carga de trabalho de enfermagem }\end{array}$ & $\begin{array}{l}\text { Sobre a carga de trabalho de enfermagem, verificou-se que a média } \\
\text { do NAS foi } 63,4 \% \text { e a média do SAPS II dos pacientes foi de } 40,7 \text {.Na } \\
\text { análise da relação do NAS com a presença de UPP, esperava-se encontrar } \\
\text { associação estatisticamente significante, no entanto isso não foi observado } \\
(p=0,702) \text {. }\end{array}$ \\
\hline Rangel \& Caliri ${ }^{19}$ & $\begin{array}{l}\text { Uso das diretrizes para tratamento da úlcera por } \\
\text { pressão por enfermeiros de um hospital geral }\end{array}$ & $\begin{array}{l}\text { Em relação às úlceras em estágio I observou-se que } 24(96 \%) \text { enfermeiros } \\
\text { relataram que sempre realizavam a mudança de decúbito e } 17 \text { (68\%) } \\
\text { sempre utilizavam colchões especiais. Verificou-se também que } \\
11(45,8 \%) \text { enfermeiros às vezes utilizavam almofadas com orifício } \\
\text { no meio. Os hidrocolóides eram utilizados às vezes por } 16(66,7 \%) \\
\text { enfermeiros, } 11 \text { (44\%) sempre utilizavam hidratantes e } 15(60 \%) \text { sempre } \\
\text { utilizavam óleos vegetais. }\end{array}$ \\
\hline Miyazaki et al. ${ }^{35}$ & $\begin{array}{l}\text { Conhecimento dos profissionais de enfermagem sobre } \\
\text { prevenção da úlcera por pressão }\end{array}$ & $\begin{array}{l}\text { Identificou-se que dos } 33 \text { itens do teste, referentes à prevenção da UPP, } \\
\text { em } 19(57,6 \%) \text { itens os participantes tiveram mais do que } 90 \% \text { de acertos, } \\
\text { em três }(9,1 \%) \text { itens entre } 70 \text { e } 89,9 \% \text { de acertos, em quatro }(12,1 \%) \text { itens } \\
\text { entre } 50 \text { e } 69,9 \% \text { de acertos e em sete }(21,2 \%) \text { itens a porcentagem de } \\
\text { acertos foi menor que } 50 \% \text {. }\end{array}$ \\
\hline Gomes et al. ${ }^{29}$ & $\begin{array}{l}\text { Fatores associados à úlcera por pressão em pacientes } \\
\text { internados nos Centros de Terapia Intensiva de Adultos }\end{array}$ & $\begin{array}{l}\text { Percebeu-se que as medidas de prevenção ainda não são adotadas de } \\
\text { forma sistemática e algumas contrariam as recomendações para uma boa } \\
\text { prática clínica, tais como a realização de massagem de conforto } \\
\text { nas proeminências ósseas e o reposicionamentos. }\end{array}$ \\
\hline Silva et al. ${ }^{22}$ & $\begin{array}{l}\text { Aplicabilidade do protocolo de prevenção de úlcera de } \\
\text { pressão em unidade de terapia intensiva }\end{array}$ & $\begin{array}{l}\text { A escala de Braden foi um instrumento bem interpretado e compreendido } \\
\text { pelos examinadores quanto ao conteúdo, a apresentação e a clareza, } \\
\text { possibilitando a utilização do protocolo com segurança e o } \\
\text { estabelecimento do diagnóstico adequado aos pacientes com risco } \\
\text { de desenvolver UPP. }\end{array}$ \\
\hline Soares et al. ${ }^{16}$ & $\begin{array}{l}\text { Análise da incidência de úlcera de pressão no } \\
\text { Hospital Metropolitano de Urgência e Emergência em } \\
\text { Ananindeua, PA }\end{array}$ & $\begin{array}{l}\text { Dentre os } 474 \text { pacientes estudados, } 17 \text { de desenvolveram úlcera de } \\
\text { pressão, com maior incidência no sexo masculino ( } 88,2 \%) \text {, em estágio II } \\
(41,2 \%) \text { e na localização sacral }(40 \%) \text {. Pacientes com mais de } 60 \text { anos de } \\
\text { idade apresentaram incidência de } 58,8 \% \text { de úlcera de pressão. } \\
\text { Ressalta-se a necessidade de criação de escalas de risco e medidas } \\
\text { preventivas para diminuir a incidência de úlceras de pressão nos } \\
\text { hospitais. }\end{array}$ \\
\hline Silva et al. ${ }^{21}$ & $\begin{array}{l}\text { Úlcera por pressão: avaliação de fatores de risco em } \\
\text { pacientes internados em um hospital universitário }\end{array}$ & $\begin{array}{l}\text { A avaliação precoce para detectar os fatores de risco para UPP } \\
\text { possibilitou adoção de medidas de prevenção de úlceras, assegurando } \\
\text { a qualidade da assistência de enfermagem. }\end{array}$ \\
\hline Stein et al. ${ }^{18}$ & $\begin{array}{l}\text { Ações dos enfermeiros na gerência do cuidado para } \\
\text { prevenção de úlceras por pressão em unidade de } \\
\text { terapia intensiva }\end{array}$ & $\begin{array}{l}\text { As principais estratégias preventivas referidas pelos enfermeiros foram } \\
\text { mudança de decúbito, exame físico diário da pele, hidratação da pele, } \\
\text { uso de coxins, suporte nutricional, uso de colchão piramidal e realização } \\
\text { de massagens de conforto. }\end{array}$ \\
\hline Dantas et al. ${ }^{24}$ & $\begin{array}{l}\text { Prática do enfermeiro intensivista no tratamento de } \\
\text { úlceras por pressão }\end{array}$ & $\begin{array}{l}\text { Para os enfermeiros, a avaliação consiste em mensurar o grau de } \\
\text { profundidade da ferida, o aspecto para, a partir da observação, escolher } \\
\text { a melhor cobertura a ser utilizada. }\end{array}$ \\
\hline
\end{tabular}

Estudo transversal realizado em um hospital geral do interior do estado de São Paulo apontou que 96,0\% dos enfermeiros preconizam a mudança de posicionamento e $68,0 \%$ relatam que utilizam colchões especiais ${ }^{19}$. Mostrou também, que o procedimento de mudança de posicionamento deve ser mantido em qualquer etapa do tratamento de UPP, uma vez que, possibilita a redução da pressão, fricção e cisalhamento ${ }^{19}$. Por evitar a compressão prolongada e a redução da irrigação sanguínea local, a mudança de posicionamento deve ser realizada de duas em duas horas, se não houver contra indicações no que diz respeito às condições gerais do paciente ${ }^{28}$. No entanto, mesmo com tais recomendações, ainda, existem serviços que apresentam falhas quanto às medidas de prevenção contra UPP ${ }^{29}$. Estudo realizado no Centro de Terapia Intensiva (CTI) de um hospital do interior paulista demonstrou que as mudanças de posicionamento são pouco utilizadas nos pacientes com e sem UPP e que $80 \%$ dos pacientes que apresentam UPP não utilizam a alternância de posição de duas em duas horas Percebeu-se que as mudanças de 
posição foram pouco realizadas tanto em pacientes com UP $(20 \%)$ como nos sem UP $(12 \%)^{28}$. O mesmo estudo, Em concordância com os resultados dos estudos apresentados nesta revisão integrativa, revela que os clientes que realizaram a mudança de posicionamento apresentaram uma incidência menor de UPP em relação aos que não foram mudados de posição ${ }^{28}$.

Outros indicadores que merecem ser citados como colaboradores para ocorrência de UPP são os fatores de risco da escala de Braden e o tempo total de internação ${ }^{24}$. Essa escala permite uma melhor avaliação de risco para o desenvolvimento de úlcera por pressão. Esta escala apresenta uma sensibilidade maior e é mais específica, oferecendo maior eficiência na avaliação, e adoção de medidas de intervenção adequadas ${ }^{20,21,28}$.

Autores afirmam que Escala de Braden é um instrumento de fácil utilização e eficiente para utilização da equipe de enfermagem na identificação dos fatores de risco de UPP em pacientes na UTI. Auxilia, de modo eficaz, os enfermeiros nas intervenções junto ao paciente, pois a escala proporciona o conhecimento do risco individual e a implementação precoce de ações de enfermagem preventivas e condizentes com este risco ${ }^{21,28,30}$.

O uso da escala de Braden possibilita, aos profissionais de saúde, melhor percepção dos fatores agressores auxiliando, deste modo, para que ocorra a implementação de medidas preventivas de úlcera por pressão, melhorando a qualidade da assistência desenvolvida ${ }^{27,30,31}$.

No que concerne à avaliação dos itens da escala de Braden, estudo realizado na UTI de um Hospital público extra-porte da cidade do Recife, mostrou que a percepção sensorial, de grande parte dos pacientes apresentou-se completamente limitada (29,0\%); e a maioria dos pacientes estudados apresentou a pele ocasionalmente úmida (50,9\%). Quanto ao grau de mobilidade física, grande parte dos pacientes estudados encontrava-se restrito aoleito $(97,3 \%)^{22}$.

É inegável que pacientes internados em UTI estão mais expostos a ocorrência de úlceras por pressão, sua prevenção constitui-se um desafio para a assistência em enfermagem e sua baixa incidência é indicativa de boa qualidade dos serviços de saúde, cabendo à equipe de enfermagem perceber a necessidade de um cuidado sistematizado ${ }^{26,30,32}$.

Contudo, dados apresentados na literatura sugerem que nem sempre a inexistência de úlcera por pressão pode indicar a qualidade do cuidado, devido à ausência de pesquisas clínicas que relatem o desaparecimento total de UPP ${ }^{32}$, mas há aquelas que demonstraram uma redução da incidência dessas úlceras, após rigorosas intervenções preventivas ${ }^{23,26,30}$. Dessa forma, cabe aos profissionais de saúde se sensibilizarem com a magnitude de tal problema.
O enfermeiro possui ações determinantes na prevenção e tratamento das úlceras por pressão. Assim, a avaliação da categoria de risco para o desenvolvimento de UPP, a individualização da assistência, a aplicação de escalas e protocolos de prevenção adequados para cada categoria de risco, a promoção da assistência e sua correta implementação, são de competência da equipe de enfermagem ${ }^{18}$. Entretanto, estudo realizado em hospital universitário na região nordeste do Brasil, sobre a prática do enfermeiro intensivista, abordou que os profissionais da enfermagem limitam o tratamento de UPP à troca de curativo e ao uso de coberturas, deixando de lado o principal fator que deve ser eliminado: a pressão ${ }^{24}$.

Dessa forma, vale ressaltar que a equipe de enfermagem deve buscar sempre melhorar seus conhecimentos, atitudes e estratégias preventivas a respeito das úlceras por pressão, destacando a importância das competências e habilidades da avaliação do enfermeiro na aplicação da assistência sistematizada, a fim de diminuir a incidência de UPP17,20,24.

\section{Os fatores de risco para UPP em pacientes internados em UTI}

Os autores abordaram os fatores de risco para UPP em pacientes internados em UTI em 5 (35,7\%) dos 14 estudos da amostra. A Tabela 3 mostra os principais resultados apresentados por esses artigos $15,18,20,34,35$.

Estudo realizado em um Hospital Universitário do município de São Paulo, incluiu dados demográficos, clínicos, de internação e a aplicação dos instrumentos Simplified Acute Physiology (SAPS II), Nursing Activities Score (NAS) e Escala de Braden. Apontou que dentre os fatores associados á úlcera por pressão encontram-se em destaque: o tempo de internação no centro de terapia intensiva superiores a 10 dias e o risco elevado na escala de Braden, com média dos escores de $12,5^{20}$. Dessa forma, a adoção de protocolos assistenciais que contemplem a magnitude desses fatores e capacitação dos profissionais de saúde pode contribuir para a prevenção desse agravo e melhoria da qualidade da assistência.

Outro estudo realizado em hospital público de UberabaMG permitiu concluir que há uma associação significativa $(p<0,05)$ entre presença de escore de risco segundo Braden e alterações neurológicas, urinárias e nutricionais nos clientes avaliados $^{15}$. A equipe multiprofissional tem a responsabilidade de programar medidas preventivas, objetivando reduzir a incidência de úlceras por pressão em pessoas internadas nas UTI, pois este é um problema de responsabilidade de todos os profissionais da saúde, especialmente da equipe enfermagem, por desempenharem atividades diretamente relacionadas ao cuidado $26,30,33,35,36$. 
Tabela 3. Principais resultados encontrados em estudos que contemplaram a categoria: "os fatores de risco para UPP em pacientes internados em UTI".

\begin{tabular}{|c|c|c|}
\hline Autor/Referência & Título & Principais resultados \\
\hline Fernandes et al. ${ }^{15}$ & $\begin{array}{l}\text { Fatores de risco e condições predisponentes para } \\
\text { úlcera de pressão em pacientes de terapia intensiva }\end{array}$ & $\begin{array}{l}\text { Os fatores intrínsecos mais frequentes nos pacientes pesquisados foram: } \\
\text { pele seca }(85,0 \%) \text {, força e/ou massa muscular diminuída }(70,0 \%) \text {, pele } \\
\text { áspera }(70,0 \%) \text {, turgor e elasticidade da pele diminuídos }(65,0 \%) \text {, } \\
\text { coordenação motora parcialmente prejudicada }(50,0 \%) \text {, edema discreto } \\
(45,0 \%) \text { e coordenação motora totalmente prejudicada }(42,5 \%) \text {. Os } \\
\text { fatores extrínsecos mais frequentes, foram: tipo de colchão inadequado } \\
(100,0 \%) \text {, posicionamento em um mesmo decúbito por mais de } 2 \text { horas } \\
(100,0 \%) \text {, força de cisalhamento/fricção }(100,0 \%) \text {, roupas de cama com } \\
\text { dobras deixando marcas no corpo }(95,0 \%) \text { e força de pressão }(95,0 \%) \text {. }\end{array}$ \\
\hline Cremasco et al. ${ }^{20}$ & $\begin{array}{l}\text { Úlcera por pressão: risco e gravidade do paciente e } \\
\text { carga de trabalho de enfermagem. }\end{array}$ & $\begin{array}{l}\text { A média do tempo de internação na UTI foi de } 13,6 \text {. Já, a média do } \\
\text { tempo de internação hospitalar foi de } 30 \text { dias. A média dos escores } \\
\text { Braden foi de } 12,5 \text {. Do total de pacientes a maioria }(55,4 \%) \text { possuía } \\
\text { escores Braden entre } 10 \text { e } 12 \text {, o que representa alto risco para UPP. } \\
\text { Pacientes com altíssimo risco para UPP, escores Braden < } 9 \text {, totalizaram } \\
10,8 \% \text {. A incidência de UPP nas UTI foi de } 31 \% \text {, sendo que a maioria } \\
(60,9 \%) \text { da ocorrência foi observada na primeira semana de internação } \\
\text { nas UTI. A gravidade dos pacientes que desenvolveram a UPP, média do } \\
\text { SAPSII } 45,87 \text {, foi estatisticamente superior ( } p=0,043) \text {, indicando que } \\
\text { quanto maior a gravidade do estado do paciente, maior a incidência } \\
\text { de UPP. }\end{array}$ \\
\hline Miyazaki et al. ${ }^{35}$ & $\begin{array}{l}\text { Conhecimento dos profissionais de enfermagem } \\
\text { sobre prevenção da úlcera por pressão. }\end{array}$ & $\begin{array}{l}\text { Observou-se que } 86,5 \% \text { dos profissionais responderam que os fatores } \\
\text { de risco para o desenvolvimento da úlcera por pressão são: imobilidade, } \\
\text { incontinência, nutrição inadequada e alteração do nível de consciência }\end{array}$ \\
\hline Gomes et al. ${ }^{29}$ & $\begin{array}{l}\text { Fatores associados à úlcera por pressão em pacientes } \\
\text { internados nos Centros de Terapia Intensiva } \\
\text { de Adultos }\end{array}$ & $\begin{array}{l}\text { Por meio da técnica de regressão logística multivariada, observou-se } \\
\text { que a presença de sepses }(\mathrm{OR}=6,04 ; \mathrm{IC} 95 \%=1,09-33,53), \\
\text { tempo de internação }>10 \text { dias }(\mathrm{OR}=7,61 ; \mathrm{IC} 95 \%=2,92-19,82) \text { e } \\
\text { risco alto e elevado na classificação da escala de Braden }(\mathrm{OR}=4,96 ; \\
\text { IC } 95 \%=1,50-16,50) \text { foram fatores independentes e significativamente } \\
\text { associados à presença de úlcera por pressão. }\end{array}$ \\
\hline Silva et al. ${ }^{22}$ & $\begin{array}{l}\text { Aplicabilidade do protocolo de prevenção de úlcera } \\
\text { de pressão em unidade de terapia intensiva. }\end{array}$ & $\begin{array}{l}\text { Fatores de risco relacionados: } 36,4 \% \text { apresentavam leve limitação } \\
\text { à percepção sensorial, 50,9\% pele ocasionalmente úmida; } 97,3 \% \\
\text { restritos ao leito; } 39,1 \% \text { muito limitados à mobilidade, } 45 \% \text { nutrição } \\
\text { provavelmente inadequada, } 61,8 \% \text { apresentaram problema quanto à } \\
\text { fricção e cisalhamento. }\end{array}$ \\
\hline Gomes et al. ${ }^{23}$ & $\begin{array}{l}\text { Avaliação de risco para úlcera por pressão em } \\
\text { pacientes críticos. }\end{array}$ & $\begin{array}{l}\text { Encontraram-se associações significantes entre: sexo/ grau de risco } \\
(p=0,016) \text {; cirurgia de grande porte/ grau de risco }(p=0,0001) \text { e } \\
\text { continência dos pacientes/mobilidade }(p=0,033) \text {. }\end{array}$ \\
\hline Silva et al. ${ }^{21}$ & $\begin{array}{l}\text { Úlcera por pressão: avaliação de fatores de risco em } \\
\text { pacientes internados em um hospital universitário. }\end{array}$ & $\begin{array}{l}\text { Houve associação significativa }(p<0,05) \text { entre escore de risco segundo } \\
\text { Braden e alterações neurológicas, urinárias, e nutricionais. }\end{array}$ \\
\hline
\end{tabular}

Em consonância com as pesquisas anteriormente citadas, estudo seccional analítico realizado em 15 hospitais públicos e privados de Belo Horizonte (MG), identificou-se por meio da técnica de regressão logística multivariada, que a presença de sepses, tempo de internação maior que 10 dias e risco alto e elevado na classificação da escala de Braden foram fatores independentes e significativamente associados à presença de úlcera por pressão ${ }^{29}$. Dentre as características clínicas do grupo que desenvolveu a lesão, os diagnósticos médicos de internação mais frequente foram as doenças respiratórias $(39,6 \%)$, cardíacas $(20,8 \%)$ e neurológicas $(17,0 \%)^{15}$.

A Unidade de Terapia Intensiva é um serviço de internação destinada a pacientes críticos, com comprometimento da percepção sensorial ou submetidos a grandes procedimentos cirúrgicos, que coloquem em risco as condições vitais. Dessa maneira, requerem atenção médica e de enfermagem permanente ${ }^{18}$. Tais condições clínicas possibilitam a esses pacientes uma maior predisposição a complicações, des- tacando-se entre elas as UPP, com repercussões na recuperação e aumento da morbimortalidade ${ }^{27}$.

Além disso, pacientes internados em UTI possuem predisposição maior para o desenvolvimento de UPP devido à dificuldade de mobilização física de modo geral, diminuindo assim, a capacidade ativa de mudança de posição no leito ${ }^{18,20}$. Os pacientes em UTI,muitas vezes,fazem também uso de anestésicos e sedativos que diminuem o nível de consciência e a sensação cutânea, causando maior vulnerabilidade a lesões de pele $\mathrm{e}^{34,37,38}$.

Sobre a localização das úlceras por pressão, estudo realizado no Hospital Universitário da Universidade de São Paulo, nas unidades de Clínica Cirúrgica (CC), Clínica Médica (CM), Unidade de Terapia Intensiva (UTI) e Unidade de Cuidados Semi- intensivos (UCSI), apontou que o local com maior incidência é a região sacra, quantificando 33,6\% dos casos; e seguida a região dos calcâneos com 24,6\% e dos glúteos, com 23,9\% $\%^{33}$. 
Deste modo, a manutenção da integridade da pele, em pacientes nos centros de terapia intensiva, torna-se um aspecto importante a ser considerado pela equipe de saúde, visto que, essas lesões são consideradas problemas graves e de custo elevado ${ }^{39}$.

\section{Fatores propulsores de assistência de enfermagem qualificada}

Em relação aos fatores propulsores de assistência de enfermagem qualificada, cinco $(35,7 \%)$ estudos abordavam esta categoria analítica. A Tabela 4 mostra os principais resultados apresentados por esses artigos ${ }^{18,19,24,35,40}$.

A úlcera por pressão configura-se como um indicador de qualidade negativa dos serviços de atenção à saúde contínua. Estudos demonstraram que o déficit de conhecimento somado a execução de práticas inadequadas pelos profissionais da saúde favorece a estagnação ou aumento quadro de úlceras $19,35,40$. Pesquisa realizada em hospital privado localizado em Natal/RN avaliando o conhecimento dos profissionais aponta um quantitativo de $100 \%$ de acertos em questões sobre o estadiamento de úlceras (I, II e IV), no entanto em questões referentes a presença de dor na úlcera por pressão em estágio II obteve um índice bem menor $(28,6 \% \text { de acertos })^{40}$.
Evidenciou-se nessa categoria analítica que níveis baixos de úlceras por pressão estão associados a uma assistência de enfermagem de qualidade; pode-se dizer que esses fatores se configuram como indicadores que avaliam a qualidade do cuidado de enfermagem nos pacientes internados, possibilitando deste modo o acompanhamento da qualidade do atendimento de enfermagem nos diferentes setores do ambiente hospitalar, podendo servir de luz para as condutas futuras da equipe ${ }^{9,31}$.

Estudo Transversal, realizado em Hospital Universitário de grande porte no Sul do Brasil, que objetivou comparar os dados notificados em um sistema de indicador de qualidade assistencial de UPP, com registros em evoluções de enfermagem nos prontuários dos pacientes, mostrou que dos $19(10 \%)$ pacientes que desenvolveram UPP categoria II ou mais, somente seis (3\%) deles foram notificados pelo sistema de indicador de qualidade assistencial, o que demonstrou uma subnotificação de UPP no indicador de qualidade assistencial ${ }^{9}$. Evidenciando, assim, que embora os indicadores de qualidade da assistência representem um avanço significativo para a qualificação do cuidado, os mesmos requerem avaliação contínua nas instituições de saúde.

Tabela 4. Principais resultados ou conclusões encontrados em estudos que contemplaram a categoria: "fatores propulsores de assistência de enfermagem qualificada".

\begin{tabular}{|c|c|c|}
\hline Autor/Referência & Título & Principais resultados \\
\hline Fernandes et al. ${ }^{41}$ & $\begin{array}{l}\text { Efeito de intervenções educativas no conhecimento } \\
\text { dos profissionais de enfermagem sobre prevenção de } \\
\text { úlceras Pressão }\end{array}$ & $\begin{array}{l}\text { Entre auxiliares e técnicos de enfermagem, o item que obteve um menor } \\
\text { índice de acertos ( } 12 \% \text { na fase pré e } 33,3 \% \text { na fase pós-intervenção } \\
\text { educativa) foi referente a necessidade de reposicionamento a cada } \\
2 \text { horas do paciente em cadeira de rodas. Observou-se aumento dos } \\
\text { acertos após intervenções educativas. }\end{array}$ \\
\hline Rangel \& Caliri ${ }^{19}$ & $\begin{array}{l}\text { Uso das diretrizes para tratamento da úlcera por } \\
\text { pressão por enfermeiros de um hospital geral }\end{array}$ & $\begin{array}{l}\text { Para as úlceras em estágio II } 13(56,5 \%) \text { enfermeiros sempre realizavam } \\
\text { a limpeza da ferida com soro fisiológico, entretanto a limpeza com água } \\
\text { e sabão era feita sempre por } 7(30,4 \%) \text { enfermeiros e por } 11(47,8 \%) \text { às } \\
\text { vezes. A utilização de óleos vegetais na ferida era realizada sempre por } \\
10(40 \%) \text { enfermeiros. O curativo de hidrocolóide nunca era utilizado } \\
\text { por } 12(57,1 \%) \text { enfermeiros. }\end{array}$ \\
\hline Miyazaki et al. ${ }^{35}$ & $\begin{array}{l}\text { Conhecimento dos profissionais de enfermagem sobre } \\
\text { prevenção da úlcera por pressão. }\end{array}$ & $\begin{array}{l}\text { Os auxiliares/técnicos de enfermagem obtiveram, em média, } 73,6 \% \\
\text { de acertos em questionário aplicado sobre UPP }(\mathrm{dp}=9,8 \%) \text { e os } \\
\text { enfermeiros } 79,4 \%(\mathrm{~d} p=8,3 \%) \text {. O teste } t \text { de Student mostrou diferença } \\
\text { estatisticamente significante }(p=0,000) \text {. A porcentagem de acertos no } \\
\text { teste dos auxiliares/técnicos de enfermagem diminuiu com o tempo de } \\
\text { formação profissional }(r=-0,170 ; p=0,009) \text {, assim como com o tempo } \\
\text { de serviço }(r=-0,125 ; p=0,049) \text {. Entretanto, no grupo dos enfermeiros, } \\
\text { a correlação encontrada entre a porcentagem de acertos e essas variáveis } \\
\text { não foi estatisticamente significante. }\end{array}$ \\
\hline Stein et al. ${ }^{18}$ & $\begin{array}{l}\text { Ações dos enfermeiros na gerência do cuidado para } \\
\text { prevenção de úlceras por pressão em unidade de } \\
\text { terapia intensiva. }\end{array}$ & $\begin{array}{l}\text { Os enfermeiros reconhecem a importância das estratégias de prevenção } \\
\text { de UPP, no entanto a sobrecarga de atividades, o estado crítico dos } \\
\text { pacientes e o alto índice de absenteísmo dificultam, muitas vezes, que } \\
\text { elas sejam implementadas no contexto investigado. }\end{array}$ \\
\hline Silva et al. ${ }^{13}$ & $\begin{array}{l}\text { Úlcera por pressão em unidade de terapia intensiva: } \\
\text { análise da incidência e lesões instaladas. }\end{array}$ & $\begin{array}{l}\text { Apesar dos investimentos em dispositivos para prevenção e tratamento } \\
\text { das úlceras por pressão, estas continuam presentes na prática e com } \\
\text { incidência significativa. Sugere-se investir em qualificação profissional, } \\
\text { bem como construir e implantar protocolos para prevenção desse agravo. }\end{array}$ \\
\hline Dantas et al. ${ }^{24}$ & $\begin{array}{l}\text { Prática do enfermeiro intensivista no tratamento de } \\
\text { úlceras por pressão }\end{array}$ & $\begin{array}{l}\text { Quando questionados sobre a participação em treinamentos que } \\
\text { abordem a temática da prevenção e tratamento de úlceras, apenas 30\% } \\
\text { dos profissionais realizaram algum tipo de atividade pertinente ao tema. }\end{array}$ \\
\hline
\end{tabular}


Diversas estratégias podem contribuir na ampliação do nível de conhecimento dos profissionais, dessa forma, torna-se relevante que se identifiquem os fatores pessoais e institucionais que possibilitam o atendimento a esta meta ${ }^{18,24}$.

Sabe-se que a metodologia utilizada pela equipe de enfermagem e seu comprometimento com o cuidado ao cliente são as bases fundamentais para a recuperação do estado de saúde do paciente. Nesse sentido, o enfermeiro deve prover de conhecimento e dispor de um método que promova a educação permanente objetivando a redução dos gastos, diminuição do tempo de internação, estabelecimento de conforto ao paciente, assim como, a produção do cuidado com qualidade ${ }^{18}$.

Dados apresentados na literatura identificam evidências que remetem aos enfermeiros a necessidade de reflexão sobre a qualidade da assistência prestada ao cliente crítico; não apenas no que tange a enfermagem, mas também à adoção de medidas que envolvam a interdisciplinaridade e multidisciplinaridade que devem sustentar o cuidado em pacientes que se encontram em estado crítico ${ }^{28,35,41,42}$.

Considera-se que uma internação de longa permanência favorece o crescimento bacteriano e a formação do biofilme, que se desenvolve nos mais diversos habitats. O que torna o paciente vulnerável a UPP, causando danos e dificultando o processo de recuperação funcional ${ }^{43}$. Os biofilmes representando um desafio terapêutico, por comprometer a cicatrização da ferida, aumentar o risco de infecção e promover mecanismos de resistência contra a ação dos antimicrobianos ${ }^{44-46}$.

Diante da complexidade da UPP, os profissionais da equipe de enfermagem devem dispor de conhecimento e sensibilidade para atuarem na resolução de problemas com tal dimensão, buscando sempre estarem atualizados sobre o tema e aptos na sistematização da assistência ${ }^{19}$.

Úlceras por Pressão é um problema de causalidade multifatorial. Na relação entre a prevenção de UPP e assistência de enfermagem em UTI o enfermeiro possui ações determinantes na prevenção e tratamento das úlceras por pressão com os indivíduos, dentre as medidas preventivas de responsabilidade da enfermagem destacou-se a mudança de decúbito.

De acordo com os resultados apresentados, percebeu-se que pacientes internados em UTI estão mais expostos ao desenvolvimento de úlceras por pressão. Cabendo à equipe de enfermagem promover a construção de uma cultura de avaliação orientada por um paradigma educativo que preconiza um olhar contínuo para a melhoria do serviço prestado.

Dentre os fatores propulsores da assistência de enfermagem percebeu - se que para qualidade do cuidado de enfermagem é necessário a realização de educação em serviço e discussões acerca da temática de UPP, para que os profissionais sejam capacitados e minimizem as lacunas na atuação nos diversos âmbitos de avaliação, prevenção e tratamento das Úlceras por Pressão, corroborando deste modo, com a redução da incidência destas em Unidades de Terapia Intensiva.

A revisão integrativa da literatura, por sua vez, possibilitou a síntese dos resultados de pesquisas relevantes, facilitando a incorporação de evidências e transferindo o conhecimento para a prática. A pesquisa pôde constatar a viabilidade clínica das escalas de Bradem e a inegável importância da mudança de posicionamento, considerada como a principal ação da equipe de enfermagem na prevenção de UPP. No entanto, ainda são necessárias novas pesquisas que continuem avaliando os riscos associados ao surgimento da UPP, bem como suas escalas de avaliação a fim de proporcionar conhecimento que possa melhorar a realidade atual sobre essa problemática.

\section{REFERÊNCIAS}

1. European Pressure Ulcer Advisory Panel and National Pressure Ulcer Advisory Panel. Prevention and treatment of pressure ulcers: quick reference guide [Internet]. Washington DC: National Pressure Ulcer Advisory Panel; 2009 [cited 2016]. Available from: http://www. epuap.org/guidelines/Final_Quick_Treatment.pdf

2. Brandão ES, Mandelbaum MHS, Santos I. A challenge in nursing care: preventing pressure ulcers in the client. J Nurs UFPE. 2012;6(8):1965-70.

3. Freitas MC, Medeiros ABF, Guedes MVC, Almeida PC, Galiza FT, Nogueira JM. Úlcera por pressão em idosos institucionalizados: análise da prevalência e fatores de risco. Rev Gaúcha Enferm. 2011;32 (1):143-50. http://dx.doi.org/10.1590/S198314472011000100019

4. Brasil. Ministério da Saúde. Portaria no 529/gm de 1 de Abril de 2013. Programa Nacional de Segurança do Paciente. Brasília: Ministério da Saúde; 2013.

5. Brasil. Agência Nacional de Vigilância Sanitária Implantação do Núcleo de Segurança do Paciente em Serviços de Saúde - Série Segurança do Paciente e Qualidade em Serviços de Saúde/Agência Nacional de Vigilância Sanitária. Brasília: Anvisa; 2014.

6. Brasil. Ministério da Saúde. Protocolo para prevenção de úlcera por pressão. Brasília: Ministério da Saúde; 2013.

7. Casaburi RP, Westin UM, Mascarenhas SHZ. Elaboração e avaliação de conteúdo educacional sobre úlceras por pressão. J Health Inform. 2012;4(Esp.):120-4.

8. Oliveira SKP, Queiroz APO, Matos DPM, Moura AF, Lima FET. Temas abordados na consulta de enfermagem: revisão integrativa da literatura. Rev Bras enferm. 2012;65(1):155-61. http://dx.doi. org/10.1590/S0034-71672012000100023

9. Santos CT, Oliveira MC, Pereira AGS, Suzuki LM, Lucena AF. Indicador de qualidade assistencial úlcera por pressão: análise de prontuário e de notificação de incidente. Rev Gaúcha Enferm. 2013;34(1): 111-8. http://dx.doi.org/10.1590/S1983-14472013000100014 
10. Simão CMF, Caliri MHL, Santos CB. Concordância entre enfermeiros quanto ao risco dos pacientes para úlcera por pressão. Acta Paul Enferm. 2013;26(1):30-5. http://dx.doi.org/10.1590/S010321002013000100006

11. Haesler E, editor, National Pressure Ulcer Advisory Panel. European Pressure Ulcer Advisory Panel and Pan Pacific Pressure Injury Alliance. Prevention and Treatment of Pressure Ulcers: quick reference guide. Cambridge Media: Perth; 2014.

12. Medeiros ABF, Lopes $C H A F$, Jorge MSB. Análise de prevenção e tratamento de úlceras por pressão propostos por enfermeiros. Rev Esc Enferm USP. 2009;43(1):223-8. http://dx.doi.org/10.1590/ S0080-62342009000100029

13. Silva MLN, Caminha RTÓ, Oliveira SHS, Diniz ERS, Oliveira JL, Neves VSN. Úlcera por pressão em unidade de terapia intensiva: análise da incidência e lesões instaladas. Rev Rene; 2013;14(5): 938-44.

14. Mendes KDS, Silveira RCCP, Galvão CM. Revisão integrativa: método de pesquisa para a incorporação de evidências na saúde e na enfermagem. Texto Contexto Enferm. 2008;17(4):758-64. http:// dx.doi.org/10.1590/S0104-07072008000400018

15. Fernandes NCS, Torres GV, Vieira D. Fatores de risco e condições predisponentes para úlcera de pressão em pacientes de terapia intensiva. Rev Eletrônica Enferm. 2008;10(3): 733-46.

16. Soares DAS, Vendramin FS, Pereira LMD, Proença PK, Marques MM. Análise da incidência de úlcera de pressão no Hospital Metropolitano de Urgência e Emergência em Ananindeua, PA. Rev Bras Cir Plást. 2011;26(4):578-81.

17. Martins DA, Soares FR. Conhecimento sobre prevenção e tratamento de úlceras de pressão entre trabalhadores de enfermagem em um hospital de Minas Gerais. Cogitare Enferm. 2008;13(1):83-7. http:// dx.doi.org/10.5380/ce.v13i1.11956

18. Stein EA, Santos JLG, Pestana AL, Guerra ST, Prochnow AG, Erdmann AL. Ações dos enfermeiros na gerência do cuidado para prevenção de úlceras por pressão em unidade de terapia intensiva. R Pesq Cuid Fundam. 2012;4(3):2605-12.

19. Rangel EML, Caliri MHL. Uso das diretrizes para tratamento da úlcera por pressão por enfermeiros de um hospital geral. Rev Eletrônica Enferm. 2009;11(1):70-7.

20. Cremasco MF, Wenzel F, Sardinha FM, Zanei SSV, Whitaker IY. Úlcera por pressão: risco e gravidade do paciente e carga de trabalho de enfermagem. Acta Paul Enferm. 2009;2(2):897-902. http://dx.doi.org/10.1590/S0103-21002009000700011

21. Silva DP, Barbosa MH, Araújo DF, Oliveira LP, Melo AF. Úlcera por pressão: avaliação de fatores de risco em pacientes internados em um hospital universitário. Rev Eletrônica Enferm. 2011;13(1): 118-23.

22. Silva EWNL, Araújo RA, Oliveira EC, Falcão VTF. Aplicabilidade do protocolo de prevenção de úlcera de pressão em unidade de terapia intensiva Rev Bras Ter Intensiva. 2010;22(2):175-85. http://dx.doi. org/10.1590/S0103-507X2010000200012

23. Gomes FSL, Bastos MAR, Matozinhos FP, Temponi HR, Meléndez GV. Avaliação de risco para úlcera por pressão em pacientes críticos. Rev Esc Enferm USP. 2010;45(2):313-8. http://dx.doi.org/10.1590/ S0080-62342011000200002
24. Dantas, ALM, Ferreira PC, Diniz KD, Medeiros ABA, Lira ALBC. Prática do enfermeiro intensivista no tratamento de úlceras por pressão. J Res: Fundam Care. 2014;6(2):716-24. http://dx.doi. org/10.9789/2175-5361.2014v6n2p716

25. Tabari L. Preparo do leito da ferida antes do tratamento cirúrgico da úlcera por pressão em indivíduos com lesão medular [dissertação]. Brasília, DF: Departamento de Enfermagem da Faculdade de Ciências da Saúde da UnB; 2014.

26. Mattia AL, Rocha AM, Barbosa MH, Guimarães MAMC, Borgato MO, Silva SRR, Freitas Filho JPA. Úlcera por Pressão em UTI: fatores de risco e medidas de prevenção. Saúde Colet. 2010;7(46):297-9.

27. Bavaresco T, Medeiros RH, Lucena AF. Implantação da escala de braden em uma unidade de terapia intensiva de um hospital universitário. Rev Gaúcha Enferm. 2011;32(4):703-10.

28. Costa IG, Caliri MHL. Validade preditiva da escala de Braden para pacientes de terapia intensiva. Acta Paul Enferm. 2011;4(6):772-7. http://dx.doi.org/10.1590/S0103-21002011000600007

29. Gomes FSL, Bastos MAR, Matozinhos FP, Temponi HR, Meléndez GV. Fatores associados a ulcera por pressão em pacientes internados nos centros de terapia intensiva de adultos. Rev Esc Enferm USP. 2010;44(4):1070-6. http://dx.doi.org/10.1590/S008062342010000400031

30. Almeida R A, Bastos RAA, Almeida FCA, Pequeno GA, Vasconcelos $J M B$, Rodrigues FA. Avaliação da utilização de protocolos na prevenção de úlceras por pressão. Ciênc. Saúde. 2012;5(2):125-31.

31. Sousa CA, Santos I, Silva LD. Aplicando recomendações da Escala de Braden e prevenindo úlceras por pressão - evidências do cuidar em enfermagem. Rev Bras Enferm. 2006;59(3):279-84. http://dx.doi. org/10.1590/S0034-71672006000300006

32. Rogenski NMB, Santos VLCG. Estudo sobre a incidência de úlceras por pressão em um hospital universitário. Rev Latino-Am Enfermagem. 2005;13(4):474-8. http://dx.doi.org/10.1590/S010411692005000400003

33. Thomas DR. Prevention and treatment of pressure ulcers. J Am Med Dir Assoc. 2006;7(1):46-59. http://dx.doi.org/10.1016/ j.jamda.2005.10.004

34. Araújo AMT, Moreira MP, Caetano JA. Avaliação de risco para úlcera por pressão em pacientes críticos. Rev Enferm UERJ. 2011;19(1): 58-63

35. Miyazaki MY, Caliri MHL, Santos CB. Conhecimento dos profissionais de enfermagem sobre prevenção da úlcera por pressão. Rev LatinoAm Enfermagem. 2010;18(6):10.

36. Araújo CRDA. Enfermagem e a utilização da escala de Braden em úlcera por pressão. Rev Enferm UERJ. 2010;18(3):359-64.

37. Dealey, C. Cuidando de feridas: um guia para enfermeiras. 3. ed. São Paulo: Atheneu; 2008.

38. Shahin ES, Dassen T, Halfens RJG: Pressure ulcer prevalence and incidence in intensive care patients: a literature review. Nurs Crit Care. 2008;13(2):71-9. http://dx.doi.org/10.1111/j.14785153.2007.00249.x

39. Araujo TM, Araujo MFM, Caetano JÁ, Galvão MTG, Damasceno MMC. Diagnósticos de enfermagem para pacientes em risco de desenvolver úlcera por pressão. Rev Bras. Enferm. 2011;64(4): 671-6. http://dx.doi.org/10.1590/S0034-71672011000400007 
40. Fernandes ML, Caliri MHL, Haas MJ. Efeito de intervenções educativas no conhecimento dos profissionais de enfermagem sobre prevenção de úlceras pressão. Acta Paul Enferm. 2008;2(2):305-11.

41. Juchem GMSSM, Falk BC, Magalhães MLR, Suzuki AMM, Midori L. Construção e implantação de dois indicadores de qualidade assistencial de enfermagem. Rev Gaúcha Enferm. 2009;30(1): 136-40.

42. Lise F, Silva LC. Prevenção de úlcera por pressão: instrumentalizando a enfermagem e orientando o familiar cuidador. Acta Sci. Health Sci. 2007;29(2):85-9.

43. Madigan MT, Martinko JM, Dunlap PV, Clark DP. Microbiologia de

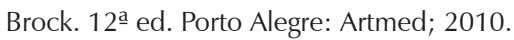

44. Smith DM, Snow DE, Rees E, Zischkau AM, Hanson JD, Wolcott RD, Sun Y, White J, Kumar S, Dowd SE. Evaluation of the bacterial diversity of pressure ulcers using bTEFAP pyrosequencing. BMC Med Genomics. 2010;3:41. http://dx.doi.org/10.1186/1755-8794-3-41

45. Romanelli M, Dini V, Barbanera S, Bertone MS. Evaluation of the efficacy and tolerability of a solution containing propyl betaine and polihexanide for wound irrigation. Skin Pharmacol Physiol. 2010;23 Suppl:41-4. http://dx.doi.org/10.1159/000318266

46. Beele H, Meuleneire F, Nahuys M, Percival SL. A prospective randomised open label study to evaluate the potential of a new silver alginate/carboxymethylcellulose antimicrobial wound dressing to promote wound healing. Int Wound J. 2010;7(4):262-70. http:// dx.doi.org/10.1111/j.1742-481X.2010.00669.x 